\title{
Static Output Feedback Controller for Structural Acoustics System
}

\author{
M. Gharib*, A. Omran*, and G. El-Bayoumi**
}

\begin{abstract}
This paper proposes designing an optimal static output feedback controller for a structural-acoustics coupled system as efficient control architecture. Modelling of sound transmission through a plate-cavity-plate system is presented. In this coupled structural acoustic system, an approximate series solution is assumed for the displacements of the plates and the pressure inside the cavity. The solution of the coupled system is obtained using Galarkin's method. The system to be modeled is consisting of a rectangular cavity with two flexible plates, one at the top of the cavity while the other at the bottom and four other fixed boundaries. Piezoelectric pair patches are considered to be bonded to the top plate, and each pair is assumed to produce a pure moment actuation. The top plate is exposed to an external pressure excitation due to a planar wave generated by a sound source mounted above the cavity. Displacements at the mid points are calculated for the upper and lower plates. In the control scheme, the controller gains have been optimally tuned using genetic algorithms. The proposed architecture is compared to the linear quadratic Gaussian state feedback controller. The two controllers are compared for the time responses. The proposed controller shows a superior performance with simple implementation requirements.
\end{abstract}

Key words: Active Control, Static Output Feedback Controller, Structural Acoustic System

\section{Introduction}

The characteristics of structure-acoustic systems for flight applications have a significant dependency on flight conditions over the entire flight envelope. These flight conditions could be defined in terms of flight speed and altitude. Designing a control, which has the capability to keep the system with acceptable behaviour regarding any change in the operating conditions, is considered as a challenging task. Gain scheduling has been effectively applied to similar control problems such as stability augmentation system (SAS) and control augmentation system (CAS) as well as it has been used for other structural applications [1]. The conceptual framework of gain schedule starts by breaking down the operating domain (flight envelopes) to a set of points. Each point has its own characteristics as functions of the flight conditions. A controller is designed for each point individually. These local controllers are scheduled to build a global one, when interpolation is employed to switch in between. The simplicity of the local control law should be sought in terms of implementation and

\footnotetext{
* Lecturer Assistant, Aerospace Engineering Department, Cairo University, Cairo, Egypt.

${ }^{* *}$ Professor, Aerospace Engineering Department, Cairo University, Cairo, Egypt
} 
computation expenses. Since, any complicated control law leads to an impractical global one, which reduces the stability and performance robustness for such control.

Linear quadratic regulator $(\mathrm{LQR})$ is the most common technique applied to structural acoustic systems [2-3]. In LQR, state feedback controller is initially computed assuming that all states are measurable. The state feedbacks control gains are computed for specific design perspectives such as eigenvalues and/or eigenvectors assignments. Although the technique has the capability to achieve a significant improvement in system behaviour, the resultant sophisticated architecture of the controller restricts the usage of such controller for a gain scheduling methodology or sensor/actuator optimal placement. In fact, the number of measurements is always less than the actual number of states. An estimator is then constructed to feed this control law by the estimated states. Such estimator is a dynamic system with the same order as the system. In this case, the control has an inherent dynamics. Most of the structural acoustic systems have a massive number of states; the number of states could be hundreds or thousands. Consequently, the control law based on LQR with estimator has the same order, which is virtually impossible to be employed for gain scheduling.

On the other hand, static output feedback (SOF) control is notable by a simple static architecture, while the measured outputs of an array of sensors are passed through a constant gain compensator matrix and back into the system as control forces. Computing this constant gain compensator matrix is considered as an optimization problem by any arbitrary performance index. The theory of an optimal SOF has been firstly presented in Ref [4]. The theory aims to optimally tune a static gain matrix to stabilizes the closed-loop system and minimize the quadratic performance given by system inputs and measurements as the case of LQR. However, unlike the LQR controller, SOF is easy to implement with more suitability for gain scheduling application and sensor/actuator placement. This simplicity brings SOF to ahead of line especially for structural systems $[\mathbf{1 , 5 - 8 ]}$, when these systems are described by hundreds of states while the number of actuator or sensor no more than ten. For example, if the system has an $N$ states vector and an $M$ inputs vector and an $P$ outputs vector, using LQR delivers a controller with $N \times N$ states and $M \times P$ transfer matrix which is computationally expensive. Instead, SOF offers the controller as a $M \times P$ static matrix.

In this paper, an optimal SOF is offered for damping plates vibration of a plate-cavity-plate system. The proposed optimal SOF is compared to LQR as a design base. The comparative study is presented in purpose to emphasize the ability of SOF to deliver a reasonable behaviour with less computation and more practical implementation. The organization of the paper proceeds as follows. In Section 2, modelling of sound transmission through a platecavity-plate system is presented. In Section 3, a brief discussion for both LQR and SOF is presented to damp the induced plates vibration due to external pressure excitation of the upper plate. The optimization technique of SOF is presented in Section 4 using genetic algorithms (GA). In Section 5, SOF is tested for a generic numerical model. The results are compared with LQR control. Finally the conclusions have been made in Section 6.

\section{Modelling of the Plate-Cavity-Plate Problem}

The system to be modelled is consisting of a rectangular cavity with two flexible plates, one at the top of the cavity while the other at the bottom and four other fixed boundaries. Piezoelectric (PZT) pair patches are considered to be bonded to the top plate (see Fig.1), and each pair is assumed to produce a pure moment actuation when an electric signal is used to excite them. The flexible plate is exposed to an external pressure excitation due to a planar 
wave generated by a sound source assumed to be mounted above the cavity. The inner dimensions of the cavity are $L_{x c}, L_{y c}$ and $L_{z c}$, in the $x, y$, and $z$ directions respectively, and the effective dimensions of the plate are $L_{x p}$ and $L_{y p}$. As shown in the schematic of the plate-cavity-plate system (Fig. 1), two coordinate systems are used to describe the system; the first one with the origin at $O_{c}$ is used for the cavity, and the second one with the origin at $O_{p}$ is used for the plates. The plate may have larger dimensions than the cavity. The two plates are set such that one of them is at the top of the cavity at $z=L_{z c}$, while the other is at the bottom of the cavity at $z=0$. Throughout the analysis, the ambient values are indicated with the subscript ( $)_{0}$. For convenience, the structural-acoustic system modelling is divided into the following subsystems: (i) the plate-cavity-plate system, (ii) the plate-piezo system, and (iii) the piezo-plate-cavity-plate system.

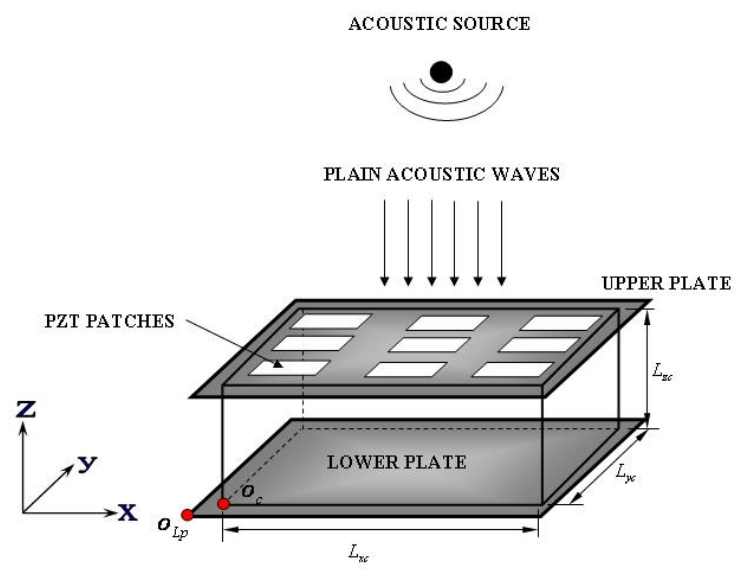

(a)

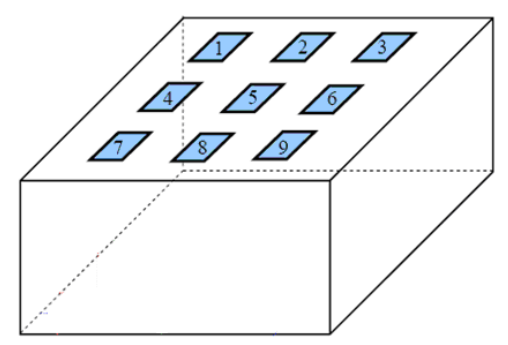

(b)

Figure 1 (a) Schematic of the plate-cavity-plate system used for the analysis model, (b) Centres locations of the actuator pairs on the upper plate

\subsection{The plate-cavity-plate system}

The two governing equations of this system are the conservation of mass equation and the conservation of momentum equation. In three-dimensional space, making use of linear approximations, the wave equation describing the sound field inside the cavity can be obtained as:

$$
\nabla^{2} P+\gamma_{c} \frac{\partial P}{\partial t}-\frac{1}{c_{o}{ }^{2}} \frac{\partial^{2} P}{\partial t^{2}}=0
$$

where $P(x, y, z ; t)$ is the air pressure inside the cavity, $\rho$ is the medium density and $\gamma_{c}$ is cavity damping coefficient. The speed of sound in a medium assuming isentropic flow is defined as [9]

$$
c=\sqrt{\frac{d P}{d \rho}}
$$

At a rigid boundary, the normal component of the air particle velocity is set to zero, and at a flexible boundary, it is set equal to the normal velocity of the flexible plate. Thus, the 
boundary conditions can be stated as:

$$
\frac{\partial P}{\partial n}=\left\{\begin{array}{cl}
0 & \text { at rigid boundary } \\
-\rho_{o} \frac{\partial^{2} w}{\partial t^{2}} & \text { at flexible boundary }
\end{array}\right.
$$

where $w(x, y ; t)$ is the normal displacement of the flexible boundary, and $n$ is the direction normal to the boundary. The pressure field inside the cavity can be expressed in the series form

$$
P(x, y, z ; t)=\sum_{i=1}^{\infty} \Phi_{i}(x, y, z) q_{i}(t)=\sum_{i=1}^{\infty} \psi_{i}(x) \varphi_{i}(y) \Gamma_{i}(z) q_{i}(t)
$$

where $\Phi_{i}(x, y, z)$ are used to describe the spatial field and $q_{i}(t)$ are used to describe the associated temporal part of the pressure response. The spatial functions $\psi_{i}(x), \varphi_{i}(y)$ and $\Gamma_{i}(z)$ are assumed to be orthogonal. Substituting Eq. (4) into Eq. (1), integrating over the volume of the cavity (Galarkin's approach), and making use of the orthogonality conditions and the boundary conditions, the cavity governing equations can be derived to have the following form

$$
\frac{1}{c_{o}^{2}} \frac{\partial^{2} q_{i}}{\partial t^{2}}+\gamma_{c} \frac{\partial q_{i}}{\partial t}+\left[k_{i}^{2}-\left.\left(\bar{\Gamma}_{i} \frac{d \bar{\Gamma}_{i}}{d z}\right)\right|_{o_{c}} ^{L_{z c}}\right] q_{i}=0
$$

where

$$
k_{i}^{2}=\left[\int_{o_{c}}^{L_{x c}}\left(\frac{d \psi_{i}}{d x}\right)^{2} d x+\int_{o_{c}}^{L_{y c}}\left(\frac{d \varphi_{i}}{d y}\right)^{2} d y+\int_{o_{c}}^{L_{z c}}\left(\frac{d \Gamma_{i}}{d z}\right)^{2} d z\right]
$$

Eq. (5) will be used later with the plate-piezo equations to satisfy the boundary condition at the flexible plate.

\subsection{The piezoelectric actuator-plate system}

The plate-piezo system is treated here as a multi-laminate system that consists of three plies in places where the piezo pair patches are bonded to the plate, and as a single ply plate otherwise. Making use of the assumptions used in earlier studies [10], the plate displacement can be described by

$$
D \nabla^{4} w+\rho_{p} h_{p} \ddot{w}+\gamma_{p} \dot{w}=p_{i n}-p_{i}-\sum_{i=1}^{k} \frac{\left(h_{p}-h_{p z t}\right) E_{p z t} d_{31}}{(1-v)} \nabla^{2} \chi_{i} V_{i}(t)
$$

where $h$ is the thickness, $D$ is the plat stiffness, $\gamma_{p}$ is the plate damping coefficient, $E$ is the modulus of elasticity, $v$ is the Poisson's ratio, $d_{31}$ is the PZT dielectric constant, and $V_{i}$ is the control voltage applied to the $i^{\text {th }}$ PZT patches. The quantity $\chi_{i}$ is unity where the $i^{\text {th }}$ PZT patch pair is present, and zero elsewhere [13]. The plate response is assumed in the series 


$$
w(x, y ; t)=\sum_{i=1}^{\infty} \alpha_{i}(x) \beta_{i}(y) \eta_{i}(t)
$$

where the $\eta_{i}(t)$ are temporal functions and the appropriate expressions for the spatial functions $\alpha_{i}(x)$ and $\beta_{i}(y)$ are obtained from the work of [11]. The upper plate only has the PZT patches and the incident pressure waves are excite it. Hence, the equations governing the two plates can be written as

$$
\begin{gathered}
D \nabla^{4} w_{U}+\rho_{p} h_{p} \ddot{w}_{U}+\gamma_{p} \dot{w}_{U}=p_{i n}-p_{U i}-\sum_{i=1}^{k} \frac{\left(h_{p}-h_{p z t}\right) E_{p z t} d_{31}}{(1-v)} \nabla^{2} \chi_{U i} V_{i}(t) \\
D \nabla^{4} w_{L}+\rho_{p} h_{p} \ddot{w}_{L}++\gamma_{p} \dot{w}_{L}=p_{i n}
\end{gathered}
$$

where the subscript ( $)_{U}$ and ()$_{L}$ are used to refer to the upper and lower plates respectively.

\subsection{The coupled piezo-plate-cavity-plate system}

In this section, the plate-cavity system is coupled with the piezoelectric actuator-plate system to obtain the governing equations for the coupled cavity subsystem. The boundary conditions at the flexible boundary are recalled from Eq. (3)

$$
\begin{aligned}
& \left.\frac{\partial P}{\partial z}\right|_{z=o_{c}}=-\rho_{o} \frac{\partial^{2} w_{L}}{\partial t^{2}} \\
& \left.\frac{\partial P}{\partial z}\right|_{z=L_{z c}}=-\rho_{o} \frac{\partial^{2} w_{U}}{\partial t^{2}}
\end{aligned}
$$

Making use of this boundary condition along with Eqs. (4) and (8), and making use of the orthogonality property, we get the following equation:

$$
\begin{aligned}
& \left.\frac{\partial \Gamma_{j}}{\partial z}\right|_{z=L_{z c}} q_{j}(t)=-\rho_{o} \sum_{i=1}^{\infty} B x_{i j}^{(c)} B y_{i j}^{(c)} \ddot{\eta}_{U}(t) \\
& \left.\frac{\partial \Gamma_{j}}{\partial z}\right|_{z=0_{o}} q_{j}(t)=-\rho_{o} \sum_{i=1}^{\infty} B x_{i j}^{(c)} B y_{i j}^{(c)} \ddot{\eta}_{L}(t)
\end{aligned}
$$

where

$$
\begin{aligned}
& B x_{i j}^{(c)}=\int_{o_{c}}^{L_{x c}} \alpha_{i}(x) \psi_{i}(x) d x \\
& B y_{i j}^{(c)}=\int_{o_{c}}^{L_{x c}} \beta_{i}(y) \varphi_{i}(y) d y
\end{aligned}
$$

After substituting Eq. (11) into Eqs. (5) and (6), we get the equation governing a pressurefield mode as follows: 


$$
\frac{1}{c_{o}{ }^{2}} \ddot{q}_{j}(t)+\gamma_{c} \dot{q}_{j}(t)+k_{j}{ }^{2} q_{j}(t)-\left(-\rho_{o} \sum_{i=1}^{\infty} B x_{i j}^{(c)} B y_{i j}^{(c)}\right)\left(\left.\Gamma_{j}\right|_{z=L_{z c}} \ddot{\eta}_{U}(t)-\left.\Gamma_{j}\right|_{z=L_{z c}} \ddot{\eta}_{L}(t)\right)=0
$$

The last terms on the left-hand side of Eq. (13) represent the structural-acoustic coupling in the system. At this stage, it is assumed that the spatial functions in Eq. (4) are given by rigidbody cavity modes; that is $[\mathbf{1 0}, \mathbf{1 2 - 1 3}]$

$$
\psi_{i}(x)=\frac{A_{i}}{\sqrt{L_{x c}}} \cos \left(\frac{l_{i} \pi x}{L_{x c}}\right), \varphi_{i}(y)=\frac{A_{i}}{\sqrt{L_{y c}}} \cos \left(\frac{m_{i} \pi y}{L_{y c}}\right), \quad \Gamma_{i}(z)=\frac{A_{i}}{\sqrt{L_{z c}}} \cos \left(\frac{n_{i} \pi z}{L_{z c}}\right)
$$

where the indices $l_{i}, m_{i}$, and $n_{i}$ are associated with the spatial functions of the $i^{\text {th }}$ rigid cavity mode, in the $x, y$, and $z$ directions, respectively. The constants $A_{i}$ are chosen to satisfy the orthogonality conditions. Using Eq. (14) the spatial function $\Gamma_{j}(z)$ at each plate will be

$$
\left(\Gamma_{j}(z)\right)_{\text {at plate }}= \begin{cases}\left(\Gamma_{j}(z)\right)_{z=L_{z c}}=\frac{(-1)^{j} A_{j}}{\sqrt{L_{z c}}} & \text { at upper plate } \\ \left(\Gamma_{j}(z)\right)_{z=0}=\frac{A_{j}}{\sqrt{L_{z c}}} & \text { at lower plate }\end{cases}
$$

Making use of Eq. (15) in Eq. (13), it is found that

$$
\begin{aligned}
\left(\frac{1}{c_{o}^{2}}\right) \ddot{q}_{j}(t) & +\gamma_{c} \dot{q}_{j}(t)+\left(\frac{l_{j}^{2} \pi^{2}}{L_{x c}^{2}}+\frac{m_{j}^{2} \pi^{2}}{L_{z c}^{2}}+\frac{n_{j}^{2} \pi^{2}}{L_{z c}^{2}}\right) q(t) \\
& +\left(\frac{(-1)^{j} A_{j}}{\sqrt{L_{z c}}} \rho_{o} \sum_{i=1}^{\infty} B x_{i j}^{(c)} B y_{i j}^{(c)}\right) \ddot{\eta}_{U}(t)-\left(\frac{A_{j}}{\sqrt{L_{z c}}} \rho_{o} \sum_{i=1}^{\infty} B x_{i j}^{(c)} B y_{i j}^{(c)}\right) \ddot{\eta}_{L}(t)=0
\end{aligned}
$$

The equations governing the plate modal amplitudes are obtained by making use of Eqs. (4), (7), (8) and (14). After making use of the orthogonality properties and boundary conditions, the equations governing the modal amplitudes of the plates are obtained as:

$$
\begin{aligned}
\rho_{p} h_{p} \ddot{\eta}_{U j}(t)+\gamma_{p} \dot{\eta}_{U j}(t)+D\left[I x_{j}+I y_{j}\right] \eta_{U j}(t)+2 D \sum_{i=1}^{\infty} I x_{i j} I y_{i j} \eta_{U i}(t) \\
=\sum_{i=1}^{\infty} \frac{(-1)^{j} A_{i}}{\sqrt{L_{z c}}} B x_{i j}^{(p)} B y_{i j}^{(p)} q_{i}(t)-\left[\int_{A_{p}} \alpha_{j} \beta_{j} p_{U i}^{s}(x, y) \mathrm{d} A_{p}\right] p_{i}^{t}(t) \\
-\sum_{i=1}^{k}\left[\int_{A_{p}} \alpha_{j} \beta_{j} \frac{\left(h_{p}+h_{p z t}\right) E_{p z t} d_{31}}{(1-v)} \nabla^{2} \chi_{U}\left(x_{i}, y_{i}\right) \mathrm{d} A_{p}\right] V_{i}(t)
\end{aligned}
$$




$$
\begin{aligned}
\rho_{p} h_{p} \ddot{\eta}_{L j}(t)+\gamma_{p} \dot{\eta}_{L j}(t)+D\left[I x_{j}+I y_{j}\right] \eta_{L j}(t)+2 D & \sum_{i=1}^{\infty} I x_{i j} I y_{i j} \eta_{L i}(t) \\
& =\sum_{i=1}^{\infty} \frac{A_{i}}{\sqrt{L_{z c}}} B x_{i j}^{(p)} B y_{i j}^{(p)} q_{i}(t)
\end{aligned}
$$

where the different spatial integrals are given by

$$
\begin{array}{ll}
B x_{i j}^{(p)}=\int_{o_{c}}^{L_{x c}} \psi_{i}(x) \alpha_{j}(x) d x & B y_{i j}^{(p)}=\int_{o_{c}}^{L_{y c}} \varphi_{i}(y) \beta_{j}(y) d y \\
I x_{j}=\int_{o_{c}}^{L_{x c}} \alpha_{j}(x) \frac{d^{4} \alpha_{j}(x)}{d x^{4}} d x & I y_{j}=\int_{o_{c}}^{L_{y c}} \beta_{j}(y) \frac{d^{4} \beta_{j}(y)}{d y^{4}} d y \\
I X_{i j}=\int_{o_{c}}^{L_{x c}} \frac{d^{2} \alpha_{i}(x)}{d x^{2}} \alpha_{j}(x) d x & I y_{i j}=\int_{o_{c}}^{L_{y c}} \frac{d^{2} \beta_{i}(y)}{d y^{2}} \beta_{j}(y) d y
\end{array}
$$

In Eq. (17), the incident pressure loading can be expressed as the product of spatial and time domain functions; that is,

$$
p_{i}(x, y ; t)=p_{i}^{s}(x, y) p_{i}^{t}(t)
$$

Now, Eqs. (16) and (17) can be represented in matrix from, after truncating the infinite number of modes to the first $M$ plate modes and $N$ acoustic modes, as follows:

$\left[\begin{array}{ccc}M_{p p}^{U} & 0 & 0 \\ M_{c p}^{U} & M_{c c} & M_{c p}^{L} \\ 0 & 0 & M_{p p}^{L}\end{array}\right]\left(\begin{array}{c}\ddot{\eta}_{U} \\ \ddot{q} \\ \ddot{\eta}_{L}\end{array}\right)+\left[\begin{array}{ccc}D_{p p}^{U} & 0 & 0 \\ 0 & D_{c c} & 0 \\ 0 & 0 & D_{p p}^{L}\end{array}\right]\left(\begin{array}{c}\dot{\eta}_{U} \\ \dot{q} \\ \dot{\eta}_{L}\end{array}\right)+\left[\begin{array}{ccc}K_{p p}^{U} & K_{p c}^{U} & 0 \\ 0 & K_{c c} & 0 \\ 0 & K_{p c}^{L} & K_{p p}^{L}\end{array}\right]\left(\begin{array}{c}\eta_{U} \\ q \\ \eta_{L}\end{array}\right)=\left[\begin{array}{cc}F_{p}^{U} & F_{V}^{U} \\ 0 & 0 \\ 0 & 0\end{array}\right]\left(\begin{array}{c}p_{i}^{t} \\ V\end{array}\right)$

In these equations $\left\{M_{p p}, K_{p p}\right\} \in \mathfrak{R}^{M \times M},\left\{M_{c c}, K_{c c}\right\} \in \mathfrak{R}^{N \times N}, M_{c p} \in \mathfrak{R}^{N \times M}, K_{p c} \in \mathfrak{R}^{M \times N}$, $F_{p} \in \mathfrak{R}^{M \times 1}$, and $F_{V} \in \mathfrak{R}^{M \times k}$. The different quantities in the above equation are given by:

$$
\begin{aligned}
& M_{p p}^{U}=M_{p p}^{L}=\operatorname{diag}\left\lfloor\rho_{p} h_{p}\right\rfloor \\
& M_{c c}^{U}=M_{c c}^{L}=\operatorname{diag}\left[\frac{1}{c_{o}^{2}}\right] \\
& M_{c p}^{U}=\rho_{o}\left[\frac{(-1)^{i} A_{i}}{\sqrt{L_{z c}}} B x_{j i}^{(c)} B y_{j i}^{(c)}\right] \\
& M_{c p}^{L}=-\rho_{o}\left[\frac{A_{i}}{\sqrt{L_{z c}}} B x_{j i}^{(c)} B y_{j i}^{(c)}\right] \\
& D_{p p}^{U}=D_{p p}^{L}=\operatorname{diag}\left\lfloor\gamma_{p}\right\rfloor
\end{aligned}
$$


$D_{c c}=\operatorname{diag}\left\lfloor\gamma_{c}\right\rfloor$

$K_{p p}^{U}=K_{p p}^{L}=2 D\left\lfloor I x_{j i} I y_{j i}\right\rfloor+D \operatorname{diag}\left\lfloor I x_{i}+I y_{i}\right\rfloor$

$K_{c c}^{U}=K_{c c}^{L}=\operatorname{diag}\left[\frac{l_{j}^{2} \pi^{2}}{L_{x c}^{2}}+\frac{m_{j}^{2} \pi^{2}}{L_{z c}^{2}}+\frac{n_{j}^{2} \pi^{2}}{L_{z c}^{2}}\right]$

$K_{p c}^{U}=\left[-\frac{(-1)^{j} A_{j}}{\sqrt{L_{z c}}} B x_{j i}^{(p)} B y_{j i}^{(p)}\right]$

$K_{p c}^{L}=\left[-\frac{A_{j}}{\sqrt{L_{z c}}} B x_{j i}^{(p)} B y_{j i}^{(p)}\right]$

$F_{p}^{U}=\left(-\int_{A_{p}} \alpha_{i} \beta_{i} p_{U i}^{s} d A_{p}\right)$

$F_{V}^{U}=\left[-\int_{A_{p}} \alpha_{i} \beta_{i} \frac{\left(h_{p}+h_{p z t}\right) E_{p z t} d_{31}}{(1-v)} \nabla^{2} \chi_{U}\left(x_{j}, y_{j}\right) \mathrm{d} A_{p}\right]$

For simplicity, the damping coefficients will be assumed constant during the numerical analysis $\left(\gamma_{c}=\gamma_{p}=0.002\right)$. The matrices $M_{c p}$ and $K_{p c}$ describe the structural-acoustic coupling, while the matrices $K_{p p}$ represent the plate stiffness matrix. Eqs. (20) represent the time-domain model developed for the system shown in Fig. 1. After determining the modal amplitudes from these equations, the plate displacements $w(x, y ; t)$ and the pressure fields inside the cavity $p(x, y, z ; t)$ can be obtained from the following relations:

$$
\left\{\begin{array}{c}
w_{U}(x, y ; t) \\
p(x, y, z ; t) \\
w_{L}(x, y ; t)
\end{array}\right\}=\left[\begin{array}{ccc}
C_{U}^{(w)}(x, y) & 0 & 0 \\
0 & C^{(p)}(x, y, z) & 0 \\
0 & 0 & C_{L}^{(w)}(x, y)
\end{array}\right]\left\{\begin{array}{c}
\eta_{U}(t) \\
q(t) \\
\eta_{L}(t)
\end{array}\right\}
$$

where

$$
\begin{gathered}
C_{U}^{(w)}=\left[\alpha_{i}(x) \beta_{i}(y)\right] \\
C^{(p)}=\left[\psi_{i}(x) \varphi_{i}(y) \Gamma_{i}(z)\right] \\
C_{L}^{(w)}=\left[\alpha_{i}(x) \beta_{i}(y)\right]
\end{gathered}
$$

\section{Control Approach}

\subsection{LQR-State Estimator Design}

The state space representation for the plate-cavity-plate system given in Section 2 is described as: 


$$
\begin{aligned}
\dot{x}(t) & =A x(t)+B_{d} d(t)+B_{u} u(t) \\
y(t) & =C x(t)
\end{aligned}
$$

where $x \in \mathfrak{R}^{N_{x}}$ is the state vector, $A \in \mathfrak{R}^{N_{x} \times N_{x}}$ is the system state dynamic matrix, $u \in \mathfrak{R}^{N_{u}}$ is the actuator command vector, $B_{u} \in \mathfrak{R}^{N_{x} \times N_{u}}$ is the control actuation matrix, $d \in \mathfrak{R}^{N_{d}}$ is the disturbance vector, $B_{d} \in \mathfrak{R}^{N_{x} \times N_{d}}$ is the disturbance actuation matrix, $y \in \mathfrak{R}^{N_{y}}$ is the sensor measurement vector, $C \in \mathfrak{R}^{N_{y} \times N_{x}}$ is the matrix relating the sensor measurement vector to the state vector. For state feedback control law, the control actuator vector $u$ is linearly related to the state vector $x$ by

$$
u(t)=-K_{x} x(t)
$$

where $K_{x} \in \Re^{N_{u} \times N_{x}}$ is the control gain matrix. The control gain matrix $K_{x}$ is computed based on specific design criteria such as pole placement or assigned eigenvector. Also, optimization is applied to compute the matrix $K_{x}$ to minimize a cost function $J(t, x, u)$. One of the most widely used and widely used method to design a full state feedback control for linear systems is the optimal LQR. Thus, the cost function $J(t, x, u)$ is defined as

$$
J(t, x, u)=\int_{0}^{\infty}\left[x^{T}(\tau) Q x(\tau)+u^{T}(\tau) R u(\tau)\right] d \tau
$$

where $Q \in \mathfrak{R}^{N_{x} \times N_{x}}$ is a symmetric positive semi-definite state weighting matrix, and $R \in \mathfrak{R}^{N_{u} \times N_{u}}$ is a symmetric positive definite control effort weighting. Since measuring the states is not possible, so estimation is the choice. If the estimate of the state vector is denoted by $\hat{x}$, then the control law in equation (25) becomes

$$
u(t)=-K_{X} \hat{x}(t)
$$

One method of estimating the state is to construct a full-order model of the plant dynamics as

$$
\dot{\hat{x}}(t)=A \hat{x}(t)+B u(t)+L(y(t)-C \hat{x}(t))
$$

where $L \in \mathfrak{R}^{N_{x} \times N_{y}}$ is the estimator gain matrix to stabilize the dynamics of estimating error defined as

$$
\dot{e}(t)=(A-L C) e(t)
$$

To success the estimation process, the estimation dynamics in equation (29) should be faster than the expected dynamics of the system. Combining both system and estimator dynamics, the overall system dynamics in the state form is

$$
\left[\begin{array}{c}
\dot{x} \\
\dot{e}
\end{array}\right]=\left[\begin{array}{cc}
A-B K & B K \\
0 & A-L C
\end{array}\right]\left[\begin{array}{l}
x \\
e
\end{array}\right]
$$




\subsection{Static Output Feedback Control}

Unlike the LQR controller, SOF assumes the control law as linear combination between the measurement vector $y$ and actuation vector $u$ as

$$
u(t)=-K_{y} y(t)
$$

where $K_{y} \in \mathfrak{R}^{N_{u} \times N_{y}}$ is the control gain matrix for SOF. This matrix is computed to stabilize the closed-loop system and minimize the quadratic performance $J(t, x, u)$. By substation from (31) to (26), the cost function $J(t, x, u)$ is then defined as

$$
J(t, x, u)=\int_{0}^{\infty} x^{T}(\tau)\left[Q+C^{T} K_{y}^{T} R C K_{y}\right] x(\tau) d \tau
$$

The cost function $J(t, x, u)$ is bounded, if the system is stable with positive definite matrix Q. The first order condition for this optimization problem is $[\mathbf{1}, \mathbf{7}]$

$$
K_{y}=-R^{-1} B_{u}^{T} S P C^{T}\left(C P C^{T}\right)^{-1}
$$

The matrices $P \in \mathfrak{R}^{N_{x} \times N_{x}}$ and $R \in \mathfrak{R}^{N_{x} \times N_{x}}$ are given by a set of coupled nonlinear matrix equations in terms of system parameters and $K_{y}$. Thus, the calculation of $K_{y}$ involves the solution of three equations including the equation (33) for $K_{y}$ and the Lyapunov equations given as $[1,7]$

$$
\begin{gathered}
\left(A-B_{u} K_{y} C\right) P+P\left(A-B_{u} K_{y} C\right)^{T}+B_{d} B_{d}^{T}=0 \\
\left(A-B_{u} K_{y} C\right)^{T} S+S\left(A-B_{u} K_{y} C\right)+Q+C^{T} K_{y}^{T} R K_{y} C=0
\end{gathered}
$$

Equations (33)-(35) are set of $3 \times N x N$ nonlinear equations. Solving this set of equations requires a special tool. Many iterative algorithms have been proposed to solve these set of equations. A detailed survey of the various computational efforts to solve optimal SOF problem is presented in [14]. Most of these algorithms incline for a local minimum points or being non-convergent. For that reason, in this paper genetic algorithm (GA) is proposed as a global search technique to solve equations (33)-(35).

\section{Optimal SOF Search Using Genetic Algorithms}

Genetic algorithm (GA) is now considered as one of the most popular optimization and search techniques. The first obvious application for the algorithm was traced back to 1962, when Holland introduced the algorithm in his work studying adaptive system [15]. The algorithm has then received an enormous exploration by Goldberg [16]. The main advantages of GA are its global optimization performance and the ease of distributing its calculations among several processors or computers as it operates on population of solutions that can be evaluated concurrently. It is a very simple method, generally applicable, not inclined to local optimization problems that arise in a multimodal search space, and no needs for special mathematical treatment. Moreover the algorithm is more applicable for the discontinuous problem, as in the case of the present study, unlike the conventional gradient-based searching algorithms. 
Basically, genetic algorithm works based on the mechanism of natural selection and evolutionary genetics. The algorithm starts by coding the variables to binary strings (chromosomes). Every chromosome has $\mathrm{n}$ genes. The gene is a binary bit by value zero or one. Three main operations control the procedure of the GA: reproduction, crossover, and mutation. Reproduction is processing to select the parent form a generation. The process is based on survival of the fittest (highest performance index). In this way, the reproduction process guides the search for best individuals (high performance index). After the individuals are selected, crossover process is then used to swap between two chromosomes by specific probabilistic decision. The crossover process generates offspring carrying mixed information from swapped parents (chromosomes). Mutation is the mechanism to prevent the algorithm from local optimal points by adding some degree of randomness. The process is performed by alternation of the gene from zero to one or from one to zero with the mutation point determined uniformly at random. The mutation rate should be consider carefully, since higher mutation rate means more number of generations are required for algorithm convergence and low mutation rate may lead to converge for local minimum. The algorithm maintains a constant size of generation by select the fittest chromosomes from parents and offsprings. The algorithm iteratively operates to converge for schema matches by some tolerance. Roughly, a genetic algorithm works as shown in Table (1). Further description of genetic algorithms can be found in Goldberg [16].

Table 1. The pseudo-code of Genetic Algorithm

\begin{tabular}{|l|}
\hline BEGIN GA \\
Make initial population at random. \\
WHILE NOT (stopping condition) DO \\
BEGIN \\
Select parents from the population. \\
Produce offspring from the selected \\
parents (crossover). \\
Mutate the individuals. \\
Extend the population adding the offspring to it. \\
Reduce the extended population. \\
END \\
Output the best individual found. \\
END GA
\end{tabular}

The algorithm listed in Table (1) is now used to solve equations (33)-(35). The step of this optimization is summarized as

- Generate an initial guessing of $K_{y}$. For efficiency of the algorithm, the steady gains of the transfer matrix of LQR $\lim _{s \rightarrow 0}[G]_{u}^{y}$ can be used as an initial value. The initial value should guarantees that the system is stable or all the poles of $\left[A-B_{u} K_{y} C\right]$ are in the left hand side. If the system is unstable for the generated $K_{y}$. The cost function is set to be zero.

- Compute $P$ from equation (34) and $S$ from equation (35). Such solution requires rewritten the two equations as a set of linear equation of both $S_{i j}$ and $P_{i j}$.

- Compute the estimated $\tilde{K}_{y}$ from equation (33). The cost function is then defined as

$$
F=\frac{1}{\varepsilon+\sum_{i=1}^{N_{y}} \sum_{j=1}^{N_{u}}\left\|K_{y}(i, j)-\tilde{K}_{y}(i, j)\right\|_{2}}
$$

where $\varepsilon$ is an arbitrarily small value prevents generating any singularity. The pseudo code in 
Table 1 keep iterates to search for maximum $\mathrm{F}$ or minimum value between the estimated and actual matrix $K_{y}$.

\section{Simulation Results}

\subsection{Structure-acoustic model}

Here, the numerical simulations obtained from the analytical model developed are presented. The numerical values used are shown in Table (2).Table 2 The numerical values used during simulation

\begin{tabular}{|lll|lll|lll|}
\hline \multicolumn{3}{|c|}{ Plate } & \multicolumn{3}{c|}{ Cavity } & \multicolumn{3}{c|}{ PZT Patch } \\
\hline$L_{x p}$ & $=24$ & inch & $L_{x c}$ & $=24$ & inch & $L_{x p z t}$ & $=2$ & inch \\
\hline$L_{y p}$ & $=18$ & inch & $L_{y c}$ & $=18$ & inch & $L_{y p z t}$ & $=1$ & inch \\
\hline$h_{p}$ & $=0.0625$ & inch & $L_{z c}$ & $=20$ & inch & $h_{p z t}$ & $=0.0125$ & inch \\
\hline$E_{p}$ & $=7.1 \times 10^{10}$ & $\mathrm{~N} / \mathrm{m}^{2}$ & $C_{o}$ & $=343$ & $\mathrm{~m} / \mathrm{sec}$ & $E_{p z t}$ & $=10.5 \times 10^{6}$ & $\mathrm{~N} / \mathrm{m}^{2}$ \\
\hline$\rho_{p}$ & $=2700$ & $\mathrm{~kg} / \mathrm{m}^{3}$ & $\rho_{o}$ & $=1.21$ & $\mathrm{~kg} / \mathrm{m}^{3}$ & $d_{31}$ & $=-247 \times 10^{-12}$ & $\mathrm{~m} / \mathrm{V}$ \\
\hline$v$ & $=0.3$ & & & & & $v$ & $=0.3$ & \\
\hline
\end{tabular}

The natural frequencies of the clamped plate have been calculated by using the following approximate formula, which is based on an energy (Raleigh) technique [11]:

$$
f_{i j}=\frac{\pi}{2} \sqrt{\frac{D}{\rho_{p} h_{p}}\left[\frac{G_{i}^{4}}{L_{x p}^{4}}+\frac{G_{j}^{4}}{L_{y p}^{4}}+\frac{2 H_{i} H_{j}}{L_{x p}^{2} L_{y p}^{2}}\right]}
$$

where the dimensionless parameters $\mathrm{G}$ and $\mathrm{H}$ are given in [11]. These parameters are functions of the indices $i$ (in the $\mathrm{x}$ direction) and $j$ (in the $\mathrm{y}$ direction) and the boundary conditions for the plate. The cavity natural frequencies are calculated through the following equation [9]:

$$
f_{i j k}=\frac{c_{o}}{2 \pi} \sqrt{\left(\frac{l_{i} \pi}{L_{x c}}\right)^{2}+\left(\frac{m_{i} \pi}{L_{y c}}\right)^{2}+\left(\frac{n_{i} \pi}{L_{z c}}\right)^{2}} \quad ; i=0,1,2, \ldots . \quad j=0,1,2, \ldots . \quad k=0,1,2, \ldots
$$

where the indices $l_{i}, m_{j}$, and $n_{k}$ are associated with the spatial functions of the $i^{\text {th }}$ rigid cavity mode in the $x, y$, and $z$ directions respectively. Using Eqs. (4) and (8), one can easily obtain the modes shapes for the plate and the cavity (see Fig. 2). The first few natural frequencies of the uncoupled and coupled system are tabulated as shown in Table (3).

Due to the complexity of the structural-acoustic coupling of this system, the effect of the stiffness coupling matrix $K_{p c}$ and the inertia coupling matrix $M_{c p}$ on the coupled natural frequencies cannot be easily realized. In Table (2), the entries of $M_{c p}$ increase the values of the first few acoustic resonance frequencies above their uncoupled values, hence, contributing a "mass reduction" effect. On the other hand, the entries of $K_{p c}$ decrease the values of the low (vibration) resonance frequencies below their uncoupled values, hence, contributing a "stiffness reduction" effect. 
Table 3 The undamped natural frequencies $(\mathrm{Hz})$ of the uncoupled and coupled system

\begin{tabular}{|c|c|c|c|c|c|c|c|c|c|}
\hline \multicolumn{7}{|c|}{ Uncoupled System } & \multicolumn{3}{|c|}{ Coupled system } \\
\hline \multicolumn{4}{|c|}{ Cavity } & \multicolumn{3}{|r|}{ Plate } & \multirow{2}{*}{\multicolumn{3}{|c|}{ Plates-Cavity-Plate System }} \\
\hline \multicolumn{3}{|c|}{ Mode } & \multirow{2}{*}{$\begin{array}{c}\begin{array}{c}\text { Closed Form } \\
\text { Formula }\end{array} \\
281.33\end{array}$} & \multicolumn{2}{|c|}{ Mode } & \multirow{2}{*}{$\begin{array}{c}\text { Approximate } \\
\text { Formula } \\
44452\end{array}$} & & & \\
\hline 1 & 0 & 0 & & 1 & 1 & & Plates & 42.75 & 43.85 \\
\hline 0 & 0 & 1 & 337.60 & 2 & 1 & 76.017 & Plates & 67.447 & 68.407 \\
\hline 0 & 1 & 0 & 375.11 & 3 & 1 & 127.66 & Plates & 132.98 & 134.11 \\
\hline 1 & 0 & 1 & 439.45 & 2 & 2 & 132.78 & Plates & 180.24 & 181.31 \\
\hline 1 & 1 & 0 & 468.89 & 3 & 2 & 181.70 & Cavity & & \\
\hline
\end{tabular}

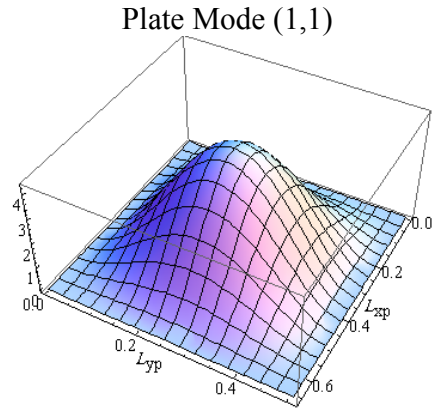

Plate Mode $(1,2)$



Cavity Mode $(1,0,0)$

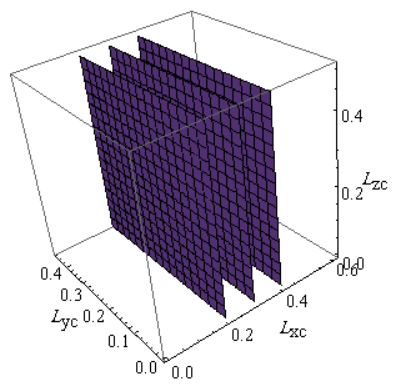

Cavity Mode $(0,1,0)$

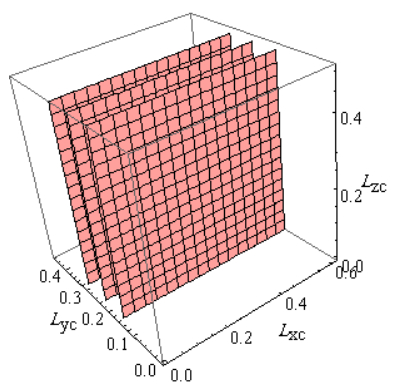

Plate Mode $(2,1)$

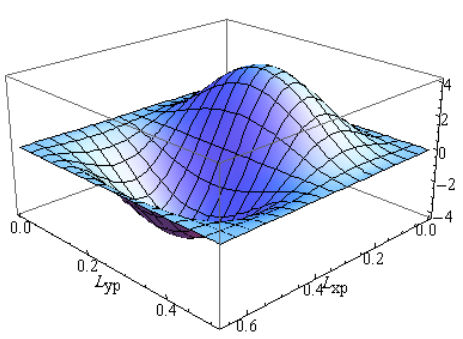

Plate Mode $(3,1)$

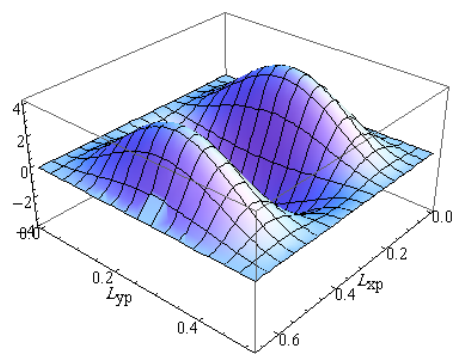

Cavity Mode $(0,0,1)$

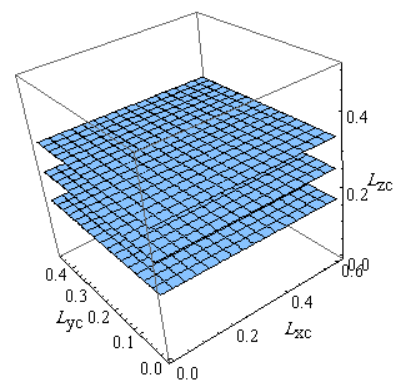

Cavity Mode $(1,0,1)$

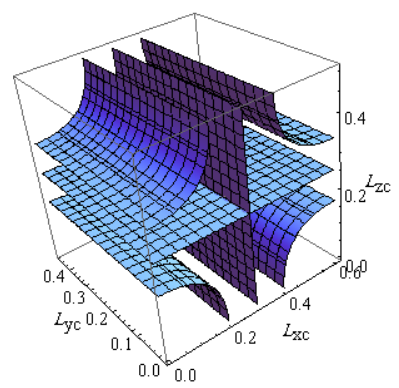

Figure 2 Plate and cavity mode shape 


\subsection{Control scheme}

For the uncompensated and compensated systems, we will use 17 plate vibration modes $(M)$ and 3 cavity acoustic modes $(N)$. The structure acoustic model outputs are nine displacements at the upper plate, nine displacements at the lower plates, and three pressures inside the cavity. Nine control actuators are assumed to be at the upper plate. The actuators and displacements positions are distributed in equal areas over the plates while the pressures are measured inside the cavity at (12", 9", 4.5”), (1.25", 4", 9.25"), and (22.75", 1.75", 1.25") in x, y and $\mathrm{z}$ directions respectively.

GA propagates based on the algorithm previously listed in sec. 4 . The mutation rate is $10 \%$. Each generation has a fixed population size 100 or no generation overlap. The algorithm is convergent for all techniques with 1000 generations. The resultant optimal controller gains in based on GA are given as

\begin{tabular}{llllllllll} 
& 0.0794 & 2.1673 & -0.6508 & 1.9377 & 3.4689 & 0.7875 & 1.5617 & -0.0916 & -3.4176 \\
-0.3919 & 1.9768 & 0.6801 & 3.9719 & -3.3297 & 3.7009 & -1.2979 & -2.873 & 4.2381 \\
-1.6105 & 2.5678 & -1.9866 & -1.2247 & 1.3101 & 3.4713 & 1.2833 & -2.895 & 1.6081 \\
-2.1648 & -1.0049 & -3.6032 & 3.8132 & 0.2503 & 0.8901 & -2.6947 & 3.3785 & 3.1538 \\
-0.0305 & 1.5006 & 1.105 & -1.6422 & -2.7485 & -4.6117 & 3.3297 & -2.2503 & -3.2491 \\
0.8852 & 3.7179 & 0.9316 & -1.7717 & 1.652 & -3.6252 & 3.1221 & -4.8022 & 1.4371 \\
-2.243 & -1.5324 & 1.5543 & 0.7387 & 2.3211 & 1.2735 & -3.3541 & 1.0488 & -2.6386 \\
3.1197 & -4.2943 & 1.906 & 1.42 & -3.8596 & 2.7021 & -1.044 & 1.7326 & 4.663 \\
-4.6044 & -2.7143 & -0.2405 & -3.9328 & -2.5263 & 2.8828 & -2.9438 & -2.2723 & 1.8181 \\
0.3114 & -0.0403 & -0.0794 & -0.0745 & 0.1624 & -0.0183 & -0.0549 & -0.0867 & -0.0183 \\
0.2601 & 0.1697 & 0.2576 & -0.0769 & 0.1282 & -0.0427 & 0.094 & 0.0085 & 0.1502 \\
-0.0037 & -0.0012 & -0.0012 & 0.0012 & -0.0037 & -0.0037 & -0.0012 & -0.0012 & -0.0012 \\
-2.4505 & -4.1062 & 3.8327 & 4.4212 & -1.0879 & -1.5372 & 0.8779 & -4.4188 & 1.2662 \\
-4.011 & 0.3919 & -4.768 & 4.221 & -4.0085 & 0.9512 & -1.8596 & -0.2454 & 1.5372 \\
-4.2063 & 3.3394 & -0.3114 & 2.9194 & 4.1209 & -2.2112 & -0.0354 & 1.2686 & -3.1148 \\
-4.2234 & 4.7778 & -3.1319 & 3.4957 & 2.3724 & 1.293 & 1.7766 & -2.9609 & 3.5446 \\
-1.3907 & -4.0037 & 3.8205 & 2.0085 & 1.1441 & 0.2137 & 2.8462 & 4.917 & 4.0061 \\
-3.0513 & -1.3053 & 3.6129 & 3.5641 & -4.6239 & 0.536 & 1.1856 & 3.4029 & -1.2833 \\
1.696 & 2.1331 & -2.348 & -1.5055 & -0.7778 & 3.0195 & -2.8901 & -3.989 & -0.8828 \\
-4.2991 & 0.3993 & 3.2662 & 0.917 & 1.1954 & -4.8755 & -3.5763 & 3.1661 & 2.4872 \\
-1.569 & 2.4335 & 4.0818 & -3.4176 & -1.5201 & -0.3382 & -0.7827 & 3.8376 & 4.9145 \\
\hline & & & & & & & &
\end{tabular}

In equation (39), the controller at the operating condition listed in Table (2) is given. Such control is a static one. At different operating conditions, other controller matrices could be computed following the same algorithm. The global controller is generated by tabulating the local controller with these flight conditions, while interpolation is employed to switch in between. On the other hand, the LQR delivers a controller with the same size; however each element is a transfer function of order $75^{\text {th }}$. Such controller is computationally inefficient and virtually impossible to be applied for gain scheduling approach. In fact, LQR provides better performance than SOF, but the performance of the SOF still has an acceptable performance.

Using the previous analysis, numerical simulation is applied to the system. The objective in the simulation is to attenuate the vibration for the upper and lower plates. Figs. 3-4 illustrate the resulting pressure response for damped sinusoidal pressure input. It is observed that the maximum amplitude and the settling time are highly reduced and the response can be tuned to a certain values by adjusting the parameters used in the control model. 


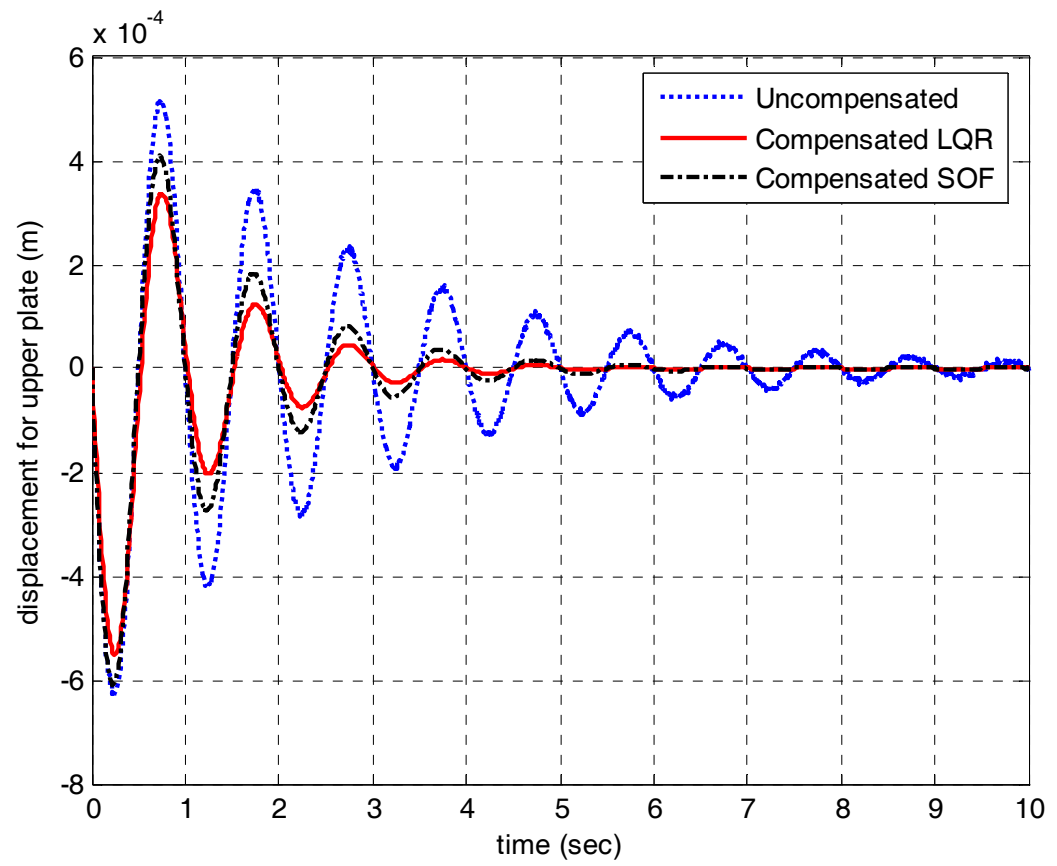

Figure 3 Uncompensated and compensated time responses due to impulse pressure input for the upper plate

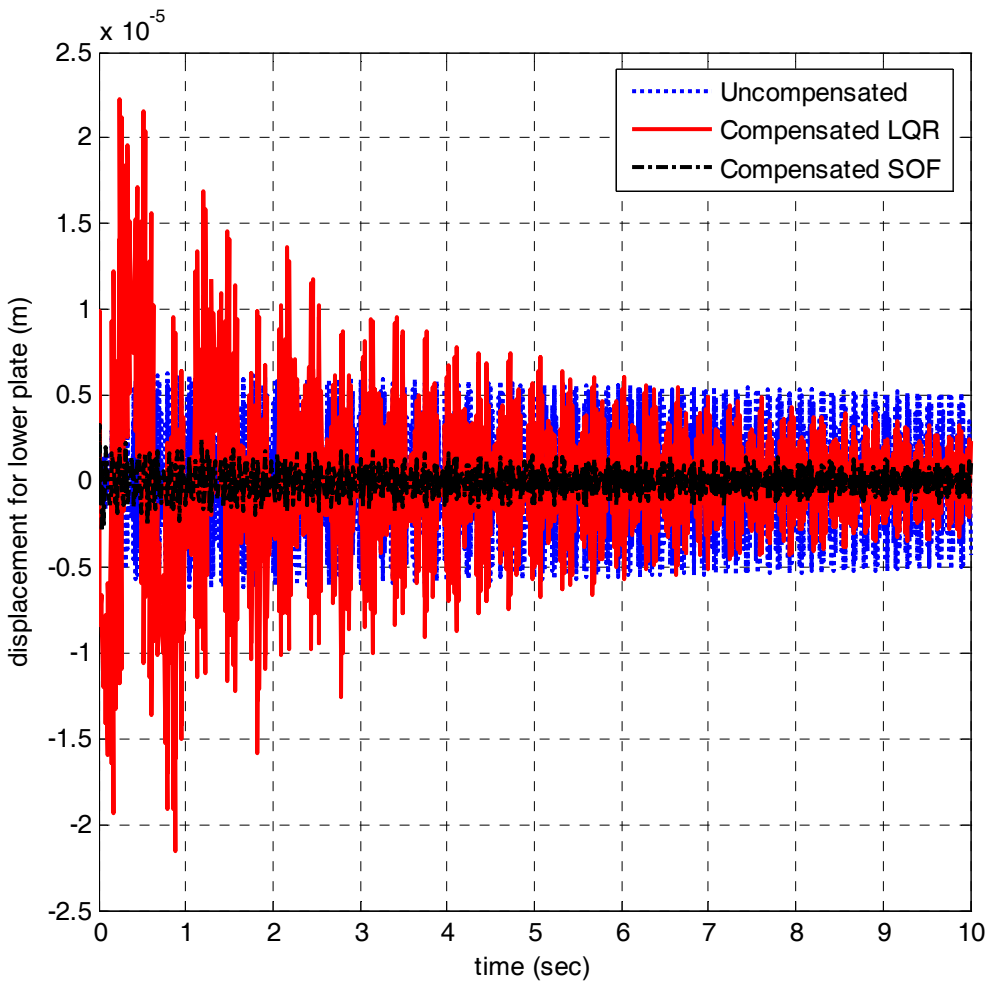

Figure 4 Uncompensated and compensated time responses due to impulse pressure input for the lower plate 


\section{Conclusions}

In this work, the development of a static output feedback controller for structure-acoustics coupled based on genetic-optimization is studied. Modelling of sound transmission through a plate-cavity-plate system is presented. The proposed controller is used in vibration damping of the plates. In the control scheme, the procedure offers a controller for practical utilization. The technique was successfully applied to one operating point. The proposed controller is a static matrix with more applicability to be scheduled. This information is unobtainable from other control methods such as a linear quadratic regulator. The piecewise interpolated could be used to extend across the flight envelope. Also, the performance of the controller is compared by the linear quadratic regulator. Based on the numerical simulations, the LQR technique has the ability to damp the vibration more than the proposed static output feedback. Such results were predicted since LQR assumes more available information than the SOF. Although the linear quadratic regulator shows better performance, the static feedback offer more practical controller. The controller is a trade-off between feasibility and performance.

\section{References}

[1] Mayuresh P., and Dewey H., "Output Feedback Control of the Nonlinear Aeroelastic Response of a Slender Wing" Journal of Guidance, Control and Dynamics, Vol. 25, No. 2, Mar.-Apr. 2002, pp. $302-30$.

[2] Gharib M. and Tawfik M., "Active Control OF Interior Noise in A Rectangular Cavity with Two Flexible Plates", 12th International Conference on Aerospace Sciences \& Aviation Technology, ASAT-12, Cairo, Egypt, 29-31 May 2007.

[3] Preumont A., and Seto K., Active control of structures, Wiley, 2008.

[4] Smith, D., Clark, L., Duke, Smith, "Optimal Transducer Placement for Output Feedback Control of Broadband Structural Acoustic Radiation," AIAA-1997-1316.

[5] Levine, W. S. and Athans, M., "On the Determination of the Optimal Constant Output Feedback Gains for Linear Multivariable Systems," IEEE Transactions on Automatic Control , Vol. AC-15, No. 1, Feb. 1970, pp. $44-48$.

[6] Waszak, M. R. and Srinathkumar, S., "Flutter Suppression for the Active Flexible Wing: A Classical Design,” Journal of Aircraft, Vol. 32, No. 1, Jan. - Feb. 1995, pp. 61 -67 .

[7] Wouter P., Oliver N., and Stephen J., "Centralized and decentralized control of structural vibration and sound radiation" Journal of the Acoustical Society of America, 119, (3), 1487-1495. March 2006.

[8] Yuan, X., Caraballo, P., Yan1, H., Ozbay, A., Serrani, J., DeBonis, H., and Myatt, M., "Reduced-order Model-based Feedback Controller Design for Subsonic Cavity Flows," 43rd AIAA Aerospace Sciences Meeting and Exhibit, 10 - 13 January 2005, Reno, Nevada.

[9] Kinsler L. E., Frey A. R., Coppens A. B., and Sanders J. V., Fundamentals of Acoustics, Fourth Edition, John Wiley and Sons, Inc., New York, (2000).

[10] Balachandran B. and Sampath A. and Park J, "Active control of interior noise in a threedimensional enclosure", Smart Materials and Structures 5, 89-97 (1996).

[11] Blevins J., Formulas for Natural Frequency and Mode Shapes, Van Nostrand Reinhold Company, (1978).

[12] Kuttruff H., Room Acoustics, 2nd Edition, Spon Press, New York, 1981.

[13] Moustafa Al-Bassyiouni and Balachandran B., "Sound transmission through a flexible plate into an enclosure: structural-acoustic model", Journal of Sound and Vibration 284, 467-486 (2005). 
[14] Makila, P. M. and Toivonen, H. T., "Computational Methods for Parametric LQ Problems - A Survey," IEEE Transactions on Automatic Control, Vol. AC-32, No. 8, August 1987, pp. $658-671$.

[15] Holland J," Adaptation in Natural and Artificial Systems," The University of Michigan Press, 1975.

[16] Goldberg, David E., "The Design of Innovation: Lessons from and for Competent Genetic Algorithms “, Boston, MA: Kluwer Academic Publishers, 2002. 